\section{Kognitive Verhaltenstherapie hilft Kindern mit Migräne}

\begin{abstract}
Die kognitive Verhaltenstherapie scheint ein guter Zugang für Kinder und Heranwachsende mit Migräne zu sein, ihre Kopfschmerztage deutlich zu reduzieren. Unterstützend wirkt das Antidepressivum Amitriptylin. Sowohl der Akut- als auch der Langzeiteffekt dieser Kombination sind überzeugend.
\end{abstract}

Rd und $2 \%$ aller Heranwachsenden leiden unter chronischer Migräne mit mindestens 15 Kopfschmerztagen pro Monat. Ob sich eine deutliche Reduktion der Kopfschmerztage durch die Behandlung mit einer kognitiven Verhaltenstherapie (CBT) plus Amitriptylin (A) erreichen lässt, sollte in einer Studie untersucht werden. Darin wurde monozentrisch die Effektivität einer Intervention in Form einer kognitiven Verhaltenstherapie plus Amitriptylin mit einer Kontrolltherapie aus Kopfschmerz-Aufklärung (HE) und Amitriptylin verglichen.

Die insgesamt 135 Kinder und Jugendlichen im Alter von 10 bis 17 Jahren do- kumentierten zunächst die Schwere ihrer Erkrankung in einem Kopfschmerztagebuch über einen Monat und wurden dann randomisiert einer Behandlung mit CBT + A oder $\mathrm{HE}+\mathrm{A}$ zugewiesen. Dabei erhielten die Patienten über acht Wochen einstündige Behandlungssitzungen und anschließend zweimal monatlich „Booster“-Sitzungen. Die Aufdosierung von Amitriptyllin erfolgte innerhalb von acht Wochen mit der Zieldosierung $1 \mathrm{mg} / \mathrm{kg} / \mathrm{d}$. Nach 20 Wochen wurde der akute Therapieerfolg und nach einem Jahr der Langzeiteffekt überprüft.

Nach 20 Wochen verzeichneten $47 \%$ der Patienten unter CBT + A vier oder weniger Kopfschmerztage pro Monat im Vergleich zu $20 \%$ der Patienten unter der $\mathrm{HE}+\mathrm{A}(\mathrm{p}=0,0011)$. Im Follow-up nach zwölf Monaten mit noch 124 teilnehmenden Patienten gaben $72 \%$ der Interventionsgruppe immer noch weniger als 4 Kopfschmerztage pro Monat im Vergleich $\mathrm{zu} 52 \%$ der Kontrollgruppe an $(\mathrm{p}=0,0249)$ und $61 \%$ der Patienten der Interventionsgruppe hatten sogar weniger als drei Kopfschmerztage pro Monat im Vergleich zu $40 \%$ unter einer Aufklärung plus Medikation ( $\mathrm{p}=0,0192)$.

Fazit: Eine kognitive Verhaltenstherapie zusätzlich zu einer antidepressiven Behandlung mit Amitriptylin reduziert langfristig die Kopfschmerztage im Vergleich zu einer Behandlung mit Antidepressivum plus Kopfschmerzerziehung.

Barbara Kreutzkamp

Kroner JW et al. Cognitive behavioral therapy plus amitriptyline for children and adolescents with chronic migraine reduced headache days to $\leq 4$ per month. Headache $2016 ; 56(4): 711-6$

\section{Zerstäubtes Methadon: effektive Schmerzkontrolle am Lebensende}

\author{
Methadon, das auf der Wangenschleimhaut zerstäubt wird, bietet eine \\ effektive und sichere Alternative zur oralen Aufnahme, wenn Patienten in \\ den letzten Lebenstagen nicht mehr schlucken können.
}

$M$ ethadon gilt als Alternative, wenn konventionelle Opioide nicht mehr vertragen werden oder der Patient nicht mehr in der Lage ist zu schlucken. Maureen Allen von der kanadischen Dalhousie University und Kollegen haben jetzt die Wirksamkeit und Handhabung einer auf die Mundschleimhaut zerstäubten Methadonlösung untersucht.

Wegen unzureichender Schmerzkontrolle oder Schwierigkeiten mit zuvor verabreichten Opioiden wurden die Studienteilnehmer während ihrer letzten Lebenstage auf Methadon umgestellt. Die Daten aus dem Palliativpflegeprogramm zu den verschiedenen transmukosalen Darreichungsformen wurden retrospektiv miteinander verglichen. Nach dem Tod der Patienten beantwor- teten Familienangehörige und Pflegepersonal Fragen zur Schmerzkontrolle und Handhabung der Methadonlösung, die sublingual, rektal oder über die Mundschleimhaut zerstäubt zugeführt wurde.

Alle 30 Studienteilnehmer behielten die Methadonmedikation bis zu ihrem Tod bei. Bevorzugt erfolgte die Applikation durch Zerstäubung auf der Mundschleimhaut (73\%), $20 \%$ erhielten das Methadon sublingual. Einer der Patienten wechselte wegen des bitteren Geschmacks zur rektalen, ein anderer auf Wunsch der Familie zur sublingualen Darreichungsform. Die durchschnittliche Dosis lag bei $37,5 \mathrm{mg} / \mathrm{d}$ über eine Dauer von vier Tagen im Median (1-12 Tage). Keiner der Patienten benötigte mehr als 1,5 ml pro Dosierung.
Alle befragten Angehörigen gaben an, die auf der Wangenschleimhaut zerstäubte Methadonlösung habe die Schmerzen effektiv kontrolliert und sei leicht zu verabreichen gewesen. Die leichte Handhabbarkeit der zerstäubten Lösung wurde von $93 \%$ des Pflegepersonals bestätigt, der sublingualen Darreichungsform von $87 \%$ und der rektalen von $60 \%$. Mit der bukkalen Schmerzkontrolle waren $73 \%$, der sublingualen $81 \%$ und der rektalen $40 \%$ der Pflegenden zufrieden.

Fazit: Die Ergebnisse zeigten, dass der Wechsel von oralem Methadon auf eine transmukosale Applikation weiterhin eine sichere und effektive Schmerzkontrolle gewährleistet. Die Zerstäubung von Methadon auf die Mundschleimhaut könne eine Alternative zur sublingualen oder rektalen Applikation darstellen.

Dr. Christine Starostzik

Allen MA et al. Effectiveness of atomized methadone on the buccal mucosa in the last days of life: An Innovative delivery route when patients can no longer swallow. J Palliat Care Med 2016; $6: 2$ 